\title{
Direct pathway cloning of the sodorifen biosynthetic gene cluster and recombinant generation of its product in E. coli
}

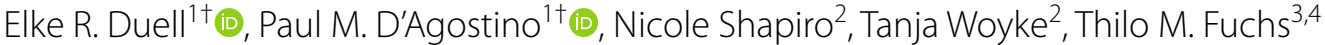 \\ and Tobias A. M. Gulder ${ }^{1,5^{*}}$ (D)
}

\begin{abstract}
Background: Serratia plymuthica WS3236 was selected for whole genome sequencing based on preliminary genetic and chemical screening indicating the presence of multiple natural product pathways. This led to the identification of a putative sodorifen biosynthetic gene cluster (BGC). The natural product sodorifen is a volatile organic compound (VOC) with an unusual polymethylated hydrocarbon bicyclic structure $\left(\mathrm{C}_{16} \mathrm{H}_{26}\right)$ produced by selected strains of $\mathrm{S}$. plymuthica. The BGC encoding sodorifen consists of four genes, two of which ( $\operatorname{sod} A$, sodB) are homologs of genes encoding enzymes of the non-mevalonate pathway and are thought to enhance the amounts of available farnesyl pyrophosphate (FPP), the precursor of sodorifen. Proceeding from FPP, only two enzymes are necessary to produce sodorifen: an S-adenosyl methionine dependent methyltransferase (SodC) with additional cyclisation activity and a terpene-cyclase (SodD). Previous analysis of S. plymuthica found sodorifen production titers are generally low and vary significantly among different producer strains. This precludes studies on the still elusive biological function of this structurally and biosynthetically fascinating bacterial terpene.
\end{abstract}

Results: Sequencing and mining of the S. plymuthica WS3236 genome revealed the presence of 38 BGCs according to antiSMASH analysis, including a putative sodorifen BGC. Further genome mining for sodorifen and sodorifen-like BGCs throughout bacteria was performed using SodC and SodD as queries and identified a total of 28 sod-like gene clusters. Using direct pathway cloning (DiPaC) we intercepted the $4.6 \mathrm{~kb}$ candidate sodorifen BGC from S. plymuthica WS3236 ( $\operatorname{sodA}-D)$ and transformed it into Escherichia coli BL21. Heterologous expression under the control of the tetracycline inducible Pteto promoter firmly linked this BGC to sodorifen production. By utilizing this newly established expression system, we increased the production yields by approximately 26 -fold when compared to the native producer. In addition, sodorifen was easily isolated in high purity by simple head-space sampling.

Conclusions: Genome mining of all available genomes within the NCBI and JGI IMG databases led to the identification of a wealth of sod-like pathways which may be responsible for producing a range of structurally unknown sodorifen analogs. Introduction of the S. plymuthica WS3236 sodorifen BGC into the fast-growing heterologous expression host E. coli with a very low VOC background led to a significant increase in both sodorifen product yield and purity compared to the native producer. By providing a reliable, high-level production system, this study sets the stage for future investigations of the biological role and function of sodorifen and for functionally unlocking the bioinformatically identified putative sod-like pathways.

\footnotetext{
*Correspondence: tobias.gulder@ch.tum.de

${ }^{\dagger}$ Elke R. Duell and Paul M. D’Agostino contributed equally to this work

1 Biosystems Chemistry, Department of Chemistry and Center for Integrated Protein Science Munich (CIPSM), Technical University

of Munich, Lichtenbergstraße 4, 85748 Garching, Germany

Full list of author information is available at the end of the article
} 
Keywords: Sodorifen, Serratia plymuthica, Terpenes, Genome mining, DiPaC, Heterologous expression

\section{Background}

Volatile organic compounds (VOCs) are lipophilic small molecules $(<300 \mathrm{Da})$ that are characterized by their high vapor pressures and low boiling points. They play important biological roles, e.g., as signaling molecules by enabling communication over long-distances and are able to facilitate cross-kingdom interactions [1-3]. Thus, understanding their ecological functions is important, for instance when looking deeper into microbial ecology or plant-rhizosphere interactions. The rhizobacterium Serratia plymuthica $4 \mathrm{Rx} 13$, for example, is emitting over 100 different VOCs which have been shown to inhibit plant and fungal growth [4-9]. The VOC profile of S. plymuthica $4 \mathrm{Rx} 13$ contains sodorifen as one major metabolite, a polymethylated hydrocarbon with an unusual bicyclo[3.2.1] octadiene skeleton (Fig. 1a) [10].

Recently, a $4.6 \mathrm{~kb}$ sodorifen (sod) BGC cluster harboring four individual genes was found to be responsible for the sodorifen production in S. plymuthica 4Rx13 (Fig. 1b) [11]. The first two genes, an isopentenyl-diphosphate delta-isomerase (IPP isomerase, $\operatorname{sod} A$ ) and a 1-deoxyD-xylulose-5-phosphate synthase (DXP synthase, $\operatorname{sod} B$ ) are homologs of genes found in the non-mevalonate pathway (MEP-pathway) and may supply additional farnesyl-pyrophosphat (FPP), the precursor of sodorifen. Notably, knock-out-mutants showed that SodB is not crucial for sodorifen emission, most likely due to the fact that one of the house-keeping enzymes of the MEPpathway provides sufficient precursor flux [11]. Expression of sodCD in Escherichia coli additionally harboring the plasmid pMEV with three MEP-pathway associated enzymes led to the production of minute amounts of sodorifen and its isomers [12]. Recent investigations in vivo and in vitro demonstrated that the SAM-dependent $C$-methyltransferase $S o d C$ is not only responsible for the addition of a methyl group to FPP, but also exhibits a surprising cyclisation activity leading to the phosphorylated version of pre-sodorifen, which is then cyclized by the terpene cyclase SodD to yield the final bicyclic compound sodorifen (Fig. 1a) [13].

Interestingly, sodorifen is produced by multiple members of the rhizobacterium family of S. plymuthica [10] suggesting an important ecological role of this specialized metabolite. Most S. plymuthica strains investigated so far emit a complex mixture of different VOCs mainly at very low concentrations [4], greatly hampering the efficient production for functional screening of individual compounds such as sodorifen. Sodorifen production can be triggered to some extend by co-cultivating S. plymuthica with the fungal pathogen Fusarium culmorum [12] and is furthermore regulated by the carbon catabolite repression system [14]. Different isolates of S. plymuthica exhibit surprisingly variable amounts of sodorifen emission, ranging from $<0.1$ to $50 \%$ of the total VOC spectrum under identical fermentation conditions [14]. Given the rather low titers of sodorifen in all natural producers, its inherent volatility and the observed complexity of VOC mixtures obtained by fermentation of S. plymuthica, studies on the functional role of this intriguing metabolite have so far been impeded. Optimized biotechnological production of sodorifen in a suitable recombinant host with low VOC background has the potential to solve this apparent supply problem thus enabling in-depth functional studies. Such an

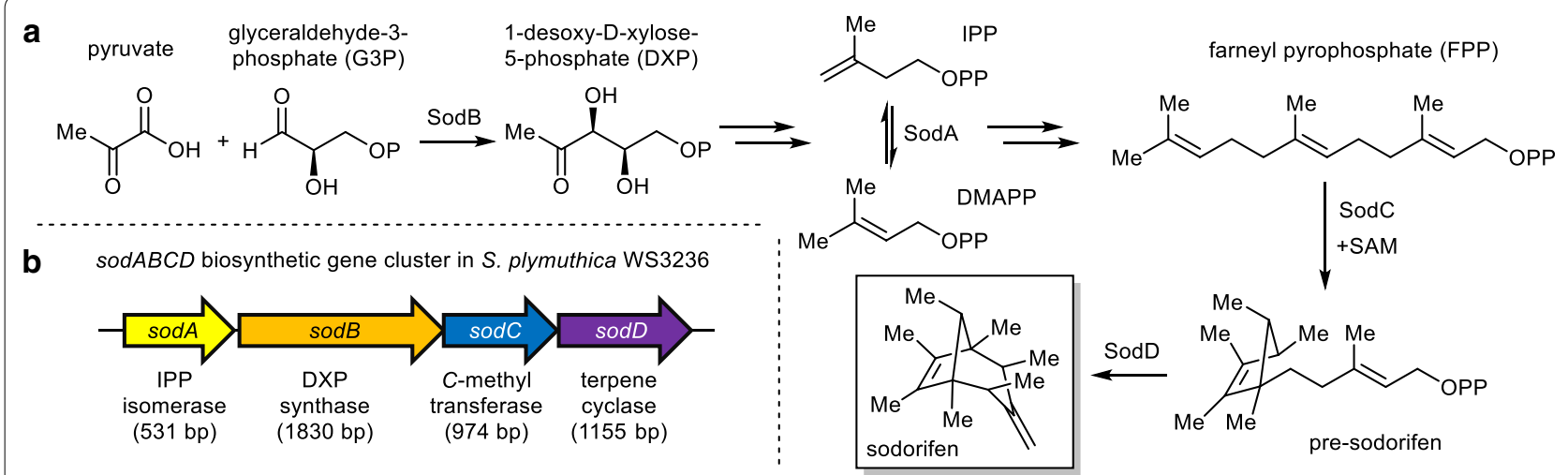

Fig. 1 a Biosynthesis of the polymethylated hydrocarbon sodorifen $\left(\mathrm{C}_{16} \mathrm{H}_{26}\right)$ from MEP-pathway-derived farnesyl pyrophosphat (FPP) via the intermediate pre-sodorifen [13]. b Sodorifen BGC from S. plymuthica WS3236 harboring an isopentenyl-diphosphate delta-isomerase sodA, a 1-deox y-D-xylulose-5-phosphate synthase sodB, a SAM-dependent C-methyltransferase sodC and a terpene cyclase sodD 
approach requires the cloning and successful heterologous, functional expression of the encoding biosynthetic gene cluster. Biosynthetic gene clusters can be captured using several in vivo methods based on recombineering, such as liner-linear homologous recombineering (LLHR) [15] or linear-circular-homologous recombineering (LCHR) [16], exonuclease combined recombination (ExoCET) [17] and Cas9-assisted targeting of chromosome segments (CATCH) [18], all utilizing the Rec/ET system [19], or using the natural recombination capability of Saccharomyces cerevisiae for transformationassociated recombination (TAR) cloning [20-22]. In addition to this, there are PCR based in vitro BGC cloning techniques such as circular polymerase extension cloning (CPEC) [23], assembly of fragment ends after PCR (AFEAP) [24] and direct pathway cloning (DiPaC) $[25,26]$. DiPaC is characterized by long-amplicon PCR combined with homologous nucleotide overhangs which allows for in vitro DNA assembly via Gibson assembly or sequence- and ligation-independent cloning (SLIC) of the BGC directly into the expression vector of choice. This enables the rapid, efficient and cheap capturing and expression of BGCs of interest in heterologous hosts such as E. coli or Streptomyces spp. [25, 26]. Within this study we present the application of $\mathrm{DiPaC}$ to functionally link a putative sodorifen biosynthetic gene cluster to sodorifen production in a recombinant host system and the optimization of the fermentative strategy to almost exclusive, high-level sodorifen production.

\section{Results}

Serratia plymuthica WS3236 was selected for WGS after preliminary genetic and chemical screening (data not shown) that pointed to the presence of natural product pathways. The resulting genome has a GC content of $55.93 \%$ and encodes a total of 5107 genes, of which 4915 (96.24\%) are protein encoding genes. The genome sequence has been deposited at GenBank SRA (BioProject ID: PRJNA442736) and IMG/MER (Accession: 2773857786) databases. To disclose the secondary metabolite potential of S. plymuthica WS3236, an in silico approach utilizing antiSMASH [27] and ClusterFinder [28] algorithms was performed. A total of 38 BGCs were identified (Additional file 1: Table S1), of which two displayed high homology to known BGCs encoding zeamine [29] (MIBiG BGC-ID: BGC0001056_c1) and sodorifen [11, 13] (MIBiG BGC-ID: BGC0001361_c1). A further seven did not show similarity to characterized pathways but belonged to well-known natural product families including non-ribosomal peptide synthase (NRPS; 4 clusters), arylpolyene-siderophore (1 cluster), polyketide synthetase/NRPS (PKS/NRPS; 1 cluster) and thiopeptide (1 cluster) biosynthesis (Additional file 1: Table S1); the remaining 29 pathways were predicted by ClusterFinder and either belonged to fatty acid, saccharide or putative pathways. Within this work, we decided to further investigate the candidate sodorifen BGC, to (1) firmly validate its small molecule product and (2) establish a reliable high-level recombinant system for sodorifen production that will facilitate future investigations into its biological functions.

To functionally link the sod candidate BGC to sodorifen production, S. plymuthica WS3236 was cultivated either in TB or succDMM, the latter being reported as the optimal sodorifen production medium for S. plymuthica [13]. Sodorifen yields were highest after day 1 and constantly decreased after this time point (Fig. 2). Interestingly, we found that cultivation in TB resulted in slightly higher sodorifen emission, which might solely be a consequence of higher growth rates and cell densities compared to the minimal succDMM medium. As neither TB nor succDMM contain glucose, the carbon catabolite repression system regulating the sod $\mathrm{BGC}$, as described by Magnus et al. [14], does not come into effect. Most importantly, all production experiments with the natural producer $S$. plymuthica WS3236 only resulted in small amounts of sodorifen relative to other VOCs produced. As purification of highly volatile organic compounds is generally difficult, we set out to construct a heterologous production system that reliably gives access to larger amounts of sodorifen, most importantly with high purity by simple head-space sampling.

For the interception of the sod BGC the DiPaC strategy was applied. An expression plasmid containing the tetracycline inducible promoter $\mathrm{Ptet}_{O}$ was chosen for heterologous expression as the use of stronger promoters such as T7 has previously been shown to frequently hamper secondary metabolite production [30]. Therefore the $4.6 \mathrm{~kb}$ sod BGC was PCR amplified in one piece using primers equipped with homologous overhangs for the vector backbone pET28b-ptetO::gfpv2 (see Fig. 3a, b). This vector carries a copy of the $g f p$ gene downstream of the multiple cloning site thus enabling the validation of transcription/translation of the complete construct by simple fluorescence detection of GFP. After linearizing the vector backbone by PCR (see Fig. 3c), the two fragments where joined using SLIC. Successful integration of the sod BGC into pET28b-ptetO::gfpv2 was verified by colony screening $\mathrm{PCR}$, analytical restriction digest and sequencing of the integration site of the resulting plasmid pET28b-ptetO::sod_gfpv2 (see Fig. 3 and Additional file 1: Figure S2).

Full transcription/translation of the sod BGC under the control of $\mathrm{Ptet}_{O}$ was confirmed by detecting green fluorescence in the presence of tetracycline, but not in the controls (see Additional file 1: Figure S3). SDS 


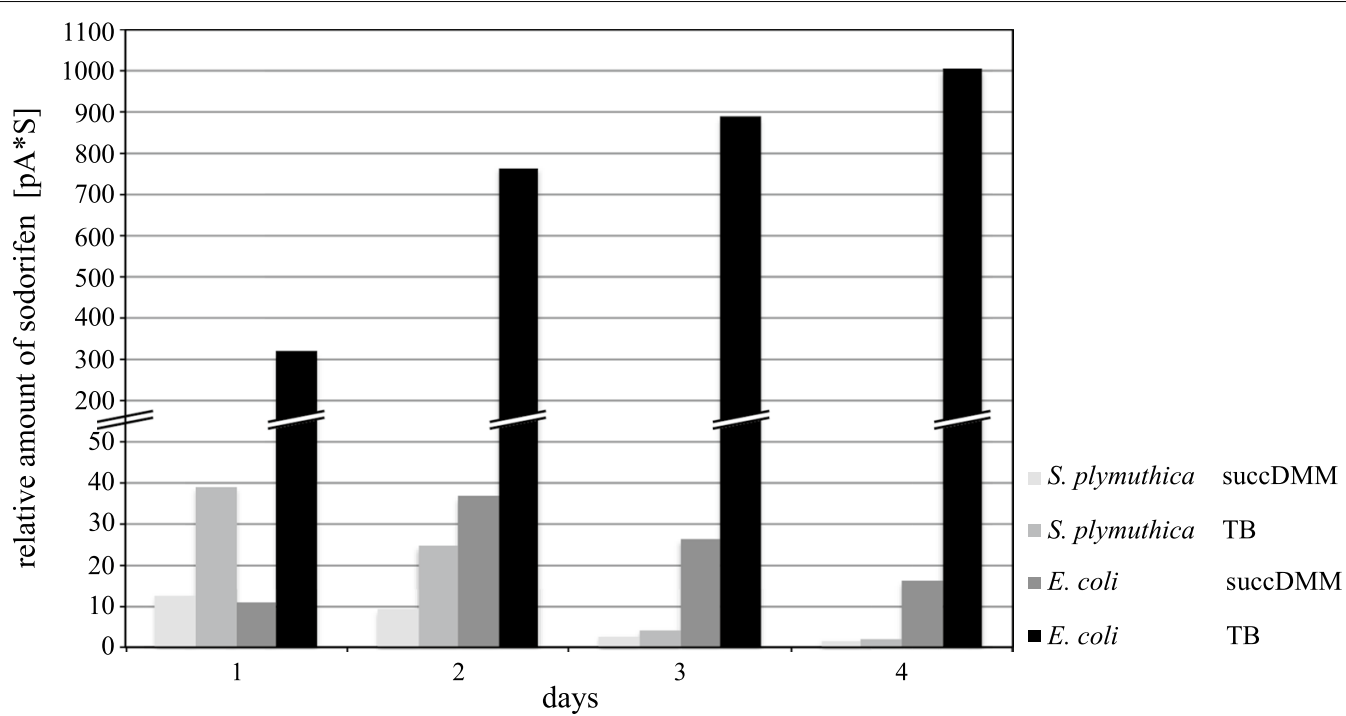

Fig. 2 Quantitative comparison of sodorifen amounts produced by S. plymuthica WS3236 and E. coli cultivated in succDMM and TB media over the course of 4 days. Yields were calculated based on peak areas PA*s measured with analytical GC. 26-fold higher sodorifen production in E. coli (TB, 4th day) can be observed compared to the best conditions tested for S. plymuthica (TB, 1st day). S. plymuthica shows a constant decrease of sodorifen production during the time course whereas for $E$. coli in TB a constant increase is observed and in succDMM a maximum is reached on the 2 nd day

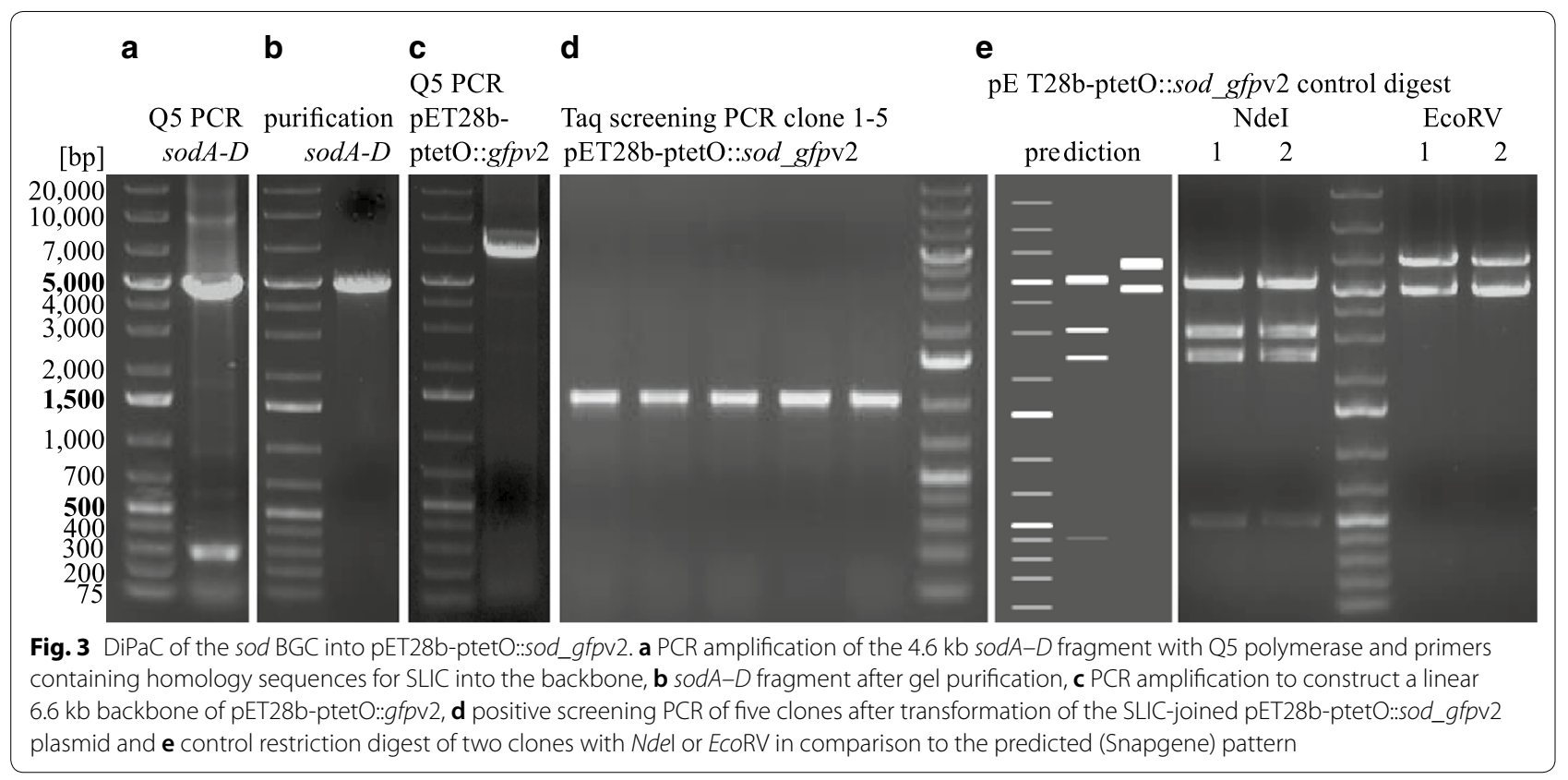

gel analysis of the whole cell protein of S. plymuthica WS3236, E. coli BL21 as well as induced versus uninduced E. coli carrying plasmid pET28b-ptetO::sod_ $g f p \mathrm{v} 2$ did not reveal any differences in the protein expression pattern (see Additional file 1: Figure S4). This clearly underlines the versatility of the applied fluorescence screening using the GFP reporter protein downstream of the sod pathway to prove successful transcription/translation of the entire construct. GCMS analysis of all VOCs emitted into the headspace of the heterologous fermentation in all three tested media revealed the accumulation of a molecule with a molecular mass of $m / z 218\left[\mathrm{M}^{+}\right]$which clearly showed the typical mass spectrum of sodorifen [10] (see Additional file 1: Figure S5). In addition, low amounts of sodorifen isomers known to also be produced by S. plymuthica 
were likewise observed [13]. As no potential sodorifen pathway intermediates were detected, apparently all terpenoid precursors available to the sodorifen biosynthetic system were fully converted to the end product by SodC and SodD.

Optimization of the heterologous production of sodorifen was tested in three different media: LB, TB and succDMM. The amounts of emitted VOC products were compared to those of S. plymuthica WS3236 under the same growth conditions. Using succDMM as cultivation medium for E. coli BL21 pET28b-ptetO::sod_gfpv2, the yield of emitted sodorifen reached a maximum on day 2 and was comparable to the production of S. plymuthica WS3236 grown in TB (cf. Fig. 2). Performing the heterologous expression in LB medium showed a maximal production on day 2 , and increased the yield about tenfold compared to succDMM (see Additional file 1: Table S4). In contrast, E. coli BL21 pET28b-ptetO::sod_gfpv2 cultivation in TB showed a tremendous boost of sodorifen emission which continuously increased with each day and revealed a 26 -fold higher production titer on day 4 when compared to the optimal production condition of the original producer (Fig. 2; see Additional file 1:
Table S4 for a complete overview of produced sodorifen amounts).

GC and GC-MS analysis revealed that S. plymuthica WS3236 produces a variety of different VOCs in comparable amounts (Fig. 4) as described previously [4]. In contrast to this result, E. coli pET28b-ptetO::sod_gfpv2 exhibits a very clean VOC profile with sodorifen as the major VOC (Fig. 4). Only in fermentations carried out in LB we found a second compound that significantly added to the spectrum of emitted substances and was subsequently identified as indole by NMR analysis, a VOC known to be produced by $E$. coli in high quantities [31, 32]. NMR measurements of the crude extract from the sampled head space of the heterologous $E$. coli fermentation in TB on day 2 showed high purity of sodorifen without any further purification (see Additional file 1: Figure S6). The relative production titers analyzed by GC-MS based on relative peak areas compared to mesitylene as an internal standard with defined concentration were nicely consistent across analyses of replicates (e.g., ranging from 232 to 318 on day one and from 1003 to 1018 at maximum production on day 3 or 4). However, isolated yields of sodorifen derived by head-space sampling
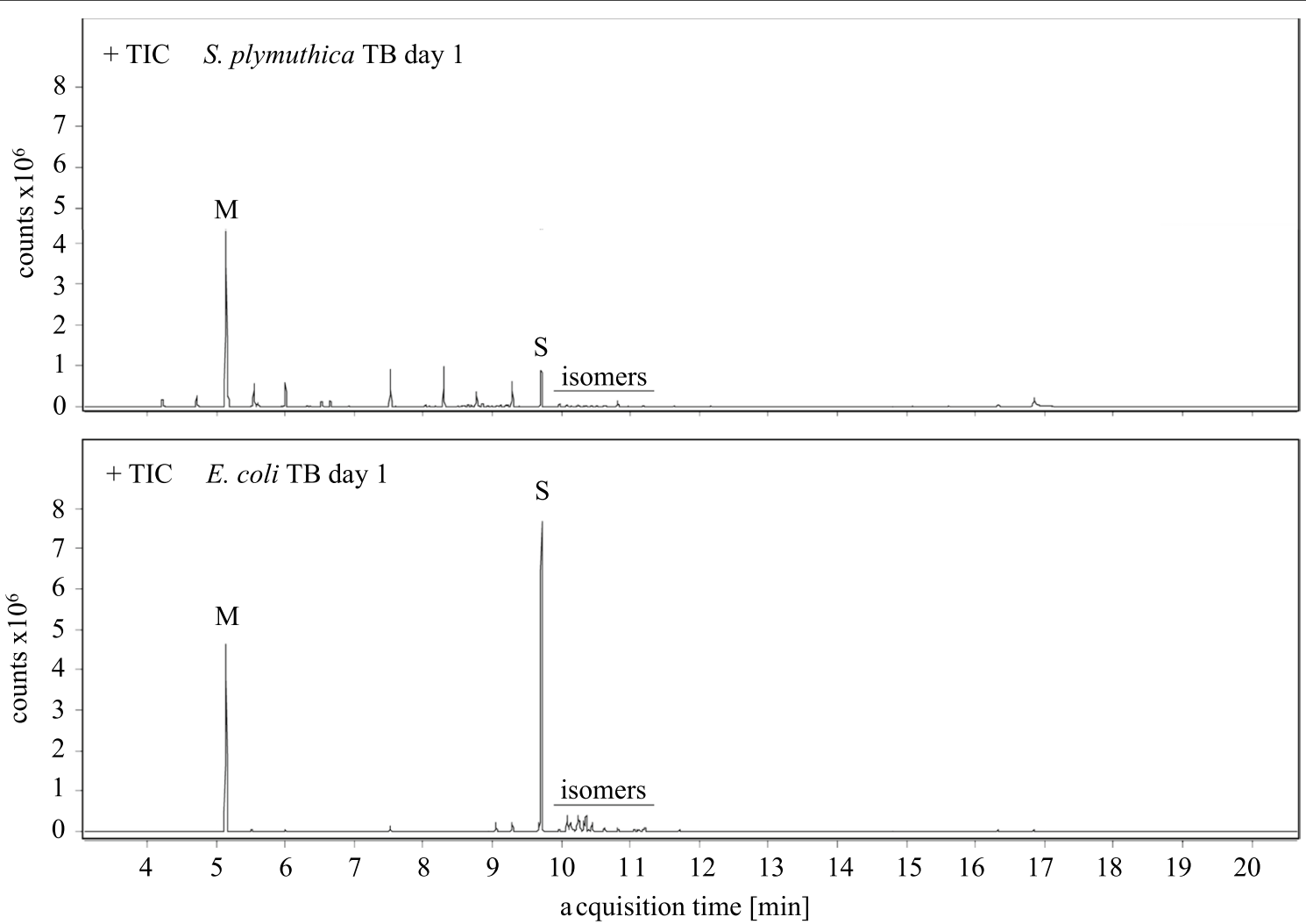

Fig. 4 GC-MS total ion chromatograms of S. plymuthica WS3236 (top) and E. coli pET28b-ptetO::sod_gfpv2 (bottom) VOCs produced in TB medium within the first $24 \mathrm{~h}$ hours of cultivation. Mesitylene (M) was added as an internal standard with a concentration of $250 \mu \mathrm{g} / \mathrm{mL}$. The amount of sodorifen (S) produced heterologously by E. coli is significantly higher combined with better purity compared to S. plymuthica 
varied more significantly (e.g., ranging from 6.4 to $7.9 \mathrm{mg}$ within $24 \mathrm{~h}$ at maximum production on days 3 or 4 ). This is a direct consequence of the volatility of sodorifen, requiring careful removal of the organic solvent under reduced pressure (pentane, used for extraction of the target compound from activated charcoal, see "Methods"). The total amount of combined isolated sodorifen headspace extract from TB fermentation over a time-course of 4 days thus ranged from 13.4 up to $25.5 \mathrm{mg}$. The developed expression system is therefore suitable for the production of sufficient amounts of sodorifen by simple head-space sampling thus establishing a reliable supply of the compound.

Given the conserved nature of the sod pathway and the unique rearranged terpene structure that is assembled following the sod biosynthetic logic, we aimed at identifying further sod-type BGCs by performing a bioinformatic screen of all publicly available microbial genomes within the NCBI and JGI IMG databases using SodC (methyltransferase) and SodD (terpene cyclase) as a query sequence, since these are essential for sodorifen biosynthesis. A total of 28 putative sod-like gene clusters were identified in S. plymuthica, Serratia sp.,
Pseudomonas chlororaphis, Pseudomonas grimontii, Pseudomonas schloroaphis, Burkholderia pyrrocinia, Burkholderia singularis and Streptomyces tsukubensis (Additional file 1: Table S2). While a sod-like gene cluster has previously been reported in P. chlororaphis O6 and Streptomyces tsukubensis NRRL18488 [11, 12], we report similar genomic regions in several other species here for the first time. Comparison of the gene synteny allowed the categorization of sod clusters into eight groups including differences in encoding one or several oxidoreductase-type enzymes, multiple methyltransferases, or multiple copies of terpene cyclase-type enzymes (Fig. 5). Based on our data, sod-like clusters are unique only at the inter-species level. Serratia sp. FS14 displayed an intriguing phylogenetic distribution, with the FS14-SodD clustering with the P. chlororaphis clade (Additional file 1: Figure S1). Of further interest was the identification of three similar terpene cyclase genes forming an operon within the $B$. singularis TSV85 sod cluster. This bioinformatic analysis clearly reveals the presence of further sod-type BGCs that are interesting targets for functional expression to obtain further unusual, structurally novel terpenoids.

Group 1: S. plymuthica WS3236

Group 1: S. plymuthica 4Rx13

Group 2: Serratia sp. FS14

Group 3: P. chlororaphis PA23

Group 4: P. schloroaphis ATCC 9446

Group 5: P. grimontii BS2976

Group 6: B. pyrrocinia Lyc2

Group 7: B. singularis TSV85

Group 8: S. tsukubensis NRRL18488
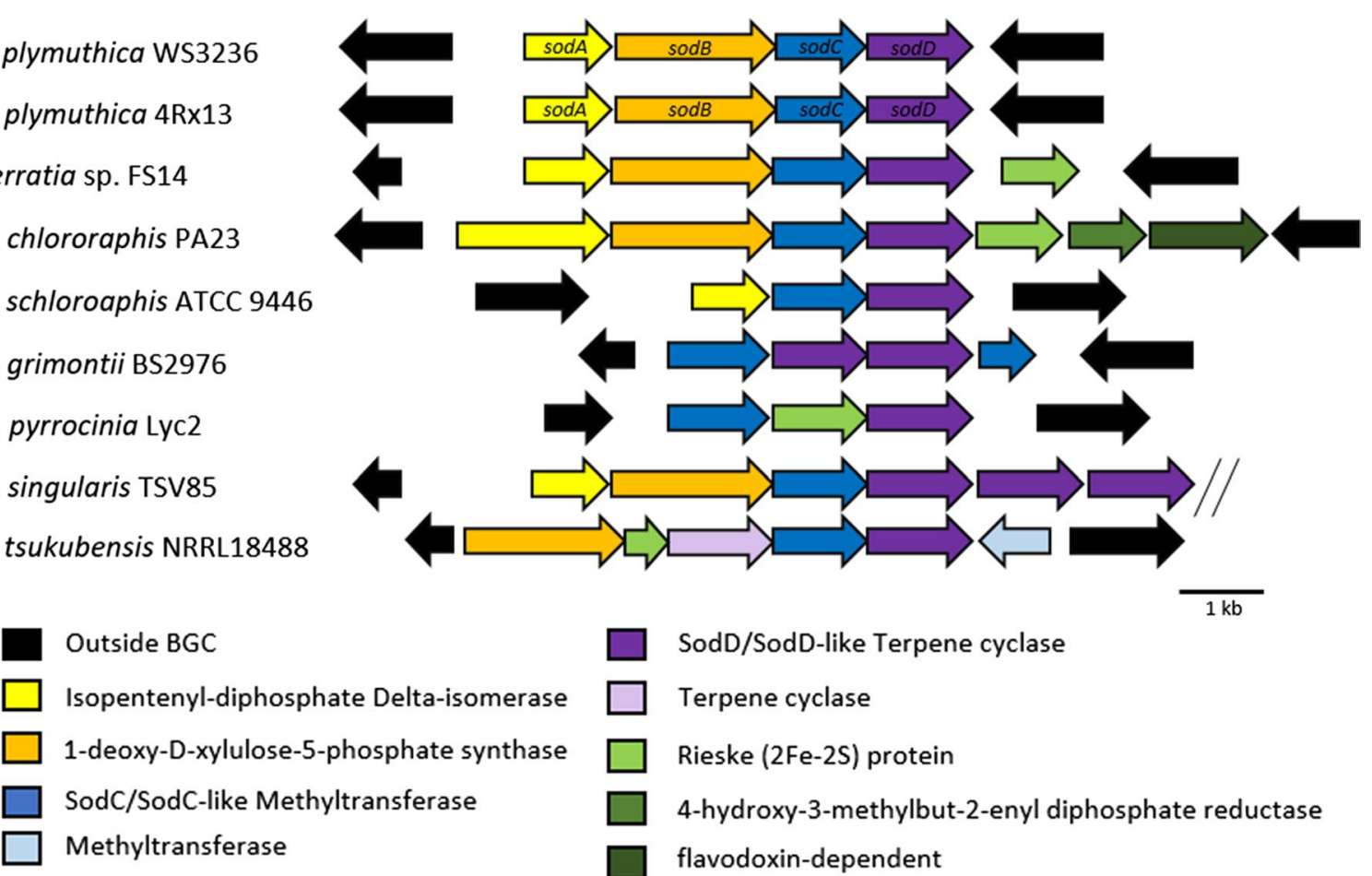

$\square$ SodD/SodD-like Terpene cyclase

Terpene cyclase

Rieske (2Fe-2S) protein

$\square$ 4-hydroxy-3-methylbut-2-enyl diphosphate reductase

Methyltransferase

flavodoxin-dependent

(E)-4-hydroxy-3-methylbut-2-enyl-diphosphate synthase

Fig. 5 Comparative analysis of sod gene clusters throughout the bacterial lineage. A total of 28 sod-like gene clusters were identified and categorized into eight groups. Groups are categorized at the inter-species level. All gene clusters except for B. pyrrocinia retained a conserved sodCD gene synteny. Double lines indicate the edge of the genome sequence contig in B. singularis TSV85 


\section{Discussion}

Serratia plymuthica is notorious for producing a wide array of VOCs. Sequencing data of the S. plymuthica WS3236 genome was consistent with other S. plymuthica strains, in particular the well characterized S. plymuthica 4Rx13 (formerly Serratia odorifera 4Rx13 [4]), which has a similar genome size and number of CDS. In silico analysis of the encoded BGCs within S. plymuthica WS3236 revealed 38 in total, with 9 BGCs belonging to well-characterized natural product families. Comparison of BGCs against $S$. plymuthica $4 \mathrm{Rx} 13$ revealed only 6 out of the 38 clusters are shared between the two organisms, highlighting the inter-species BGC variability in the genus Serratia.

Most S. plymuthica strains investigated so far emit a complex mixture of a large number of different VOCs, most of them produced at very low levels [4], making the functional screening of individual compounds such as sodorifen almost impossible. Schmidt et al. [12] recently developed a successful $E$. coli based system harboring the sodorifen methyltransferase and the terpene cyclase as well as an additional mevalonate pathway-expression plasmid encoding a mevalonate kinase, a phosphomevalonate kinase and a mevalonate pyrophosphate decarboxylase to increase the presence of farnesyl pyrophosphate, the precursor of sodorifen, but were only able to produce low amounts of sodorifen [12]. To further improve the yield of heterologously producible sodorifen, we thus decided to use the entire natural sodorifen production gene cassette under the control of an inducible promoter. We selected the tetracycline-induced promoter Ptet ${ }_{O}$, which has successfully been utilized in the heterologous expression of multiple natural product BGCs [25, 26, 33]. This expression system was also successful in this study and allowed for a constant sodorifen biosynthesis in amounts significantly higher than the natural producer. With this approach, we were able to increase the amount of emitted sodorifen up to 26-fold compared to the optimal production conditions of the original producer $S$. plymuthica WS3236. In addition, we obtained sodorifen in high purity by head-space sampling, thus providing an efficient system for sodorifen production, which sets the stage for further investigations into the biological function of this interesting compound.

Within this work, we furthermore investigated the occurrence of additional candidate sodorifen-type BGCs in bacteria. In silico screening of published genomes led to the identification of $28 \mathrm{sod}$ and sodlike gene clusters throughout bacteria, which is a significant increase on previously reported numbers [11, 12]. Intriguingly, while Serratia sp. FS14 was isolated from plants, similar to other sodorifen producing bacteria, its genome is more closely related to non-plant associated Serratia marcescens [34], but still encodes a sod gene cluster. The sod cluster of Serratia sp. FS14 differed significantly compared to other Serratia, with a much lower sequence identity ( 63\% FS14 vs WS3236 compared to $\sim 96 \% 4 \mathrm{Rx} 13$ vs WS3236) and the presence of a Rieske (2Fe-2S) protein that is similar to those in P. chlororaphis (58\%) and B. pyrrocinia (56\%). The phylogenetic clustering of FS14-SodD with homologous proteins from $P$. chlororaphis (Additional file 1: Figure S1) may be the first hint that organisms outside of $S$. plymuthica species are capable of producing sodorifen or sodorifen analogs.

Based on gene synteny analysis, we were able to categorize the sod-like clusters into eight groups, which mostly separated at the species level. Differences between these sod-like clusters include the presence of one or several oxidoreductase-type enzymes, multiple methyltransferases, or multiple copies of terpene cyclase-type enzymes. This suggests that there are significant modifications to the sodorifen backbone in order to produce a range of analogues, which could be a significant source of novel unusual bacterial terpenes.

\section{Conclusions}

Genome sequencing and bioinformatic analysis revealed that there is a significant BGC diversity within different S. plymuthica species. Comparison and phylogenetic analysis of SodD of Serratia sp. FS14 indicates this pathway shares some homology with $P$. chlororaphis, raising interesting evolutionary questions about sodorifen biosynthesis. Our in silico analysis furthermore revealed the presence of a diversity of sod-type BGCs across phylogenetically diverse bacteria. Significant genetic variability (e.g., additional methyltransferases, terpene cyclases and oxidoreductase enzymes) between these pathways indicates that there likely is a plethora of unique and structurally intriguing sodorifen analogs awaiting discovery. Using $\mathrm{DiPaC}$, we quickly integrated the native four gene containing sodorifen cluster sod $A B C D$ into a vector under the control of one single promoter upstream of $\operatorname{sod} A$, thereby mimicking the transcription setting in the original producer. This ensures that the relevant enzymes of the MEP-pathway SodA and SodB are overproduced synchronously with SodC and SodD, which finally transform SAM and FPP into sodorifen. By screening a variety of different culture conditions, sodorifen production was increased up to 26-fold compared to the original producer, accompanied by significantly improved purity in the crude extract. The results of this work pave the way for future studies investigating the biological role and function of sodorifen. 


\section{Methods}

\section{Bacterial culturing, strains and plasmids}

Bacterial strains and plasmids generated in this study are listed in Table 1. S. plymuthica WS3236 was obtained from the Weihenstephaner collection of microorganisms (Chair of Microbial Ecology, Technical University of Munich Freising, Germany). S. plymuthica WS3236 was cultivated in nutrient broth (Carl Roth, Germany) at $28{ }^{\circ} \mathrm{C}$ (liquid cultures) or $30{ }^{\circ} \mathrm{C}$ (agar plates). Liquid cultures were incubated while shaking at $200 \mathrm{rpm}$. Under routine culture conditions unless otherwise specified, $E$. coli strains were grown in lysogeny broth (LB) medium (Carl Roth, Germany) supplemented with $50 \mu \mathrm{g} / \mathrm{mL}$ kanamycin.

\section{Genomic DNA extraction, whole genome sequencing (WGS) and assembly}

Extraction of high-molecular weight genomic DNA was performed as described by Micallef [35] and Greunke et al. [25] with few alterations. After culturing, cells were placed at $4{ }^{\circ} \mathrm{C}$ for $24 \mathrm{~h}$ to stop cell division and DNA replication prior to DNA extraction. After centrifugation (10 min at $10,000 \times g)$, the cell pellet was resuspended in $5 \mathrm{~mL}$ lysis buffer (25 mM EDTA, 0.3 M sucrose, $25 \mathrm{mM}$ Tris- $\mathrm{HCl}[\mathrm{pH} 7.5])$. The cells were freeze-thawed three times (liquid nitrogen $/ 50{ }^{\circ} \mathrm{C}$ ) to promote cell lysis. Lysozyme (Sigma-Aldrich) was added to a final concentration of $1 \mathrm{mg} / \mathrm{mL}$ and RNase (Carl Roth, Germany) to a final concentration of $10 \mu \mathrm{g} / \mathrm{mL}$ followed by an incubation at $37{ }^{\circ} \mathrm{C}$ for $60 \mathrm{~min}$. Proteinase $\mathrm{K}$ (Amresco, USA) was added to a final concentration of $0.5 \mathrm{mg} / \mathrm{mL}$ followed by the addition of SDS to a final concentration of $1 \%$ $(\mathrm{w} / \mathrm{v})$. The cell mixture was incubated at $37^{\circ} \mathrm{C}$ for $30 \mathrm{~min}$ followed by $55{ }^{\circ} \mathrm{C}$ for $30 \mathrm{~min}$. $\mathrm{NaCl}$ was added to a final concentration of $1.3 \mathrm{M}$, and cetyltrimethylammonium bromide (CTAB) [10\% (w/v) CTAB (Sigma-Aldrich) in $0.7 \mathrm{M} \mathrm{NaCl}$ ] to a final concentration of $1 \%(\mathrm{v} / \mathrm{v}) \mathrm{CTAB}$ solution, with the mixture incubated at $65{ }^{\circ} \mathrm{C}$ for $10 \mathrm{~min}$. A total of 1 vol of chloroform:isoamyl alcohol (24:1) was added to the cell lysis solution, mixed by inversion and incubated on ice while shaking for $30 \mathrm{~min}$. The aqueous phase was removed and phenol-chloroform-isoamylalcohol (25:24:1) extraction was performed twice before the addition of $0.6 \mathrm{vol}$ of isopropanol. The DNA cell pellet was washed with ice cold $70 \%$ ethanol and finally dissolved in $0.1 \times$ TE buffer $(1 \mathrm{mM}$ Tris- $\mathrm{HCl}, 0.1 \mathrm{mM}$ EDTA [pH 8.0]). The quality of gDNA was analyzed by gel electrophoresis using $0.7 \%(\mathrm{w} / \mathrm{v})$ agarose gels and quantified with a P330 NanoPhotometer (Implen, Germany).

The whole genome of S. plymuthica WS3236 was generated at the DOE Joint Genome Institute (JGI) using the Pacific Biosciences (PacBio) sequencing technology [36]. A > 10 kb PacBio SMRTbell ${ }^{\mathrm{TM}}$ library was constructed and sequenced on the PacBio RS2 platform, which generated 78,182 filtered subreads totaling $307,584,001 \mathrm{bp}$. The raw reads were assembled using HGAP (smrtanalysis/2.3.0 p5, HGAP 3) [37]. The final draft assembly contained 1 contig in 1 scaffold, totaling 5,349,225 bp in size. The input read coverage was $45.2 \times$. The genome sequencing QC report can be found as Additional file 2.

\section{Bioinformatic and phylogenetic analysis}

In silico analysis of the BGCs present in the S. plymuthica WS3236 genome was performed using AntiSMASH (Version 4) [27]. The presence of the sod cluster throughout all available genome sequences against the NCBI and JGI IMG databases (as of October 2018) was searched using the S. plymuthica SodC (methyltransferase; locus_tag: Ga0236286_4400) and SodD (terpene cyclase; locus_tag: Ga0236286_4399) as query sequences. BlastP analysis with these query sequences was performed against the NCBI non-redundant and JGI Integrated Microbial Genomes System [38] databases. All hits consisting of adjacent methyltransferase and terpene cyclase genes were categorized as possible sod-type clusters. Phylogenetic analysis was performed using the Phylogeny.fr online tool with 'one click' analysis [39, 40]. All

\section{Table 1 Bacterial strains and plasmids used in this study}

\begin{tabular}{|c|c|c|}
\hline & Description & Reference or source \\
\hline \multicolumn{3}{|l|}{ Strains } \\
\hline Escherichia coli DH5a & Host strain for cloning & NEB \\
\hline Escherichia coli BL21 & Heterologous expression strain & NEB \\
\hline Serratia plymuthica WS3236 & Native producer of sodorifen & ZIEL Institute Culture Collection \\
\hline \multicolumn{3}{|l|}{ Plasmids } \\
\hline $\begin{array}{l}\text { pET28b-ptetO-gfpv2 } \\
(6029 \text { bp) }\end{array}$ & $\begin{array}{l}\text { Tetracycline inducible expression plasmid, ColE1, Kan }{ }^{\mathrm{R}} \text {, gfp reporter gene downstream } \\
\text { of promotor }\end{array}$ & This study \\
\hline $\begin{array}{l}\text { pET28b-ptetO::sod_gfpv2 } \\
(11,124 \text { bp) }\end{array}$ & pET28b-ptetO_gfpv2 with sodABCD cloned as single fragment between Ptet 0 and gfp & This study \\
\hline
\end{tabular}


sequences were stored and visualized using the Geneious Software Package.

\section{Direct pathway cloning of the sod cluster}

The $4.6 \mathrm{~kb}$ sod cluster of S. plymuthica WS3236 was cloned in one piece into pET28b-ptetO using SLIC-mediated $\mathrm{DiPaC}$ [26]. Linearized PCR amplicons of both the vector backbone and the sodABCD fragment were generated using PCR with Q5 polymerase (NEB) under standard conditions with 50 ng gDNA per $25 \mu \mathrm{L}$ reaction setup (see Additional file 1: Table S3 for primer sequences and chapter 2.1 for PCR setup). At the $5^{\prime}$ end of the cluster specific primer pair, 22 bp homology sequences consistent with the terminal region of the PCR generated vector were added. After purification, the fragments were assembled with T4-DNA polymerase and $8 \mu \mathrm{L}$ of the reaction mixture was chemically transformed into $E$. coli DH5 $\alpha$. Positive pET28b-ptetO::sod_gfp 2 clones were selected by Taq screening PCR with primers binding sodD and the T7 terminator (see Additional file 1: Table S3 for primer sequences and chapter 2.2 for PCR setup) and confirmed using restriction digest and Sanger sequencing of the integration sites.

\section{Bacterial fermentation for sodorifen production}

All fermentations were performed in $3 \mathrm{~L}$ scale using a BIOSTAT A plus fermenter (type 8843812, Sartorius Stedim Biotech) and the volatile compounds were collected at the gas outlet with a filter consisting of $0.5 \mathrm{~g}$ pure activated charcoal between two VitraPOR glass filters (porosity 00, ROBU, Germany) in a plastic centrifuge column (Pierce $5 \mathrm{~mL}$, Thermo Scientific, Germany). $3 \mathrm{~L}$ cultures were inoculated 1:50 from overnight precultures grown in LB medium and $300 \mu \mathrm{L}$ of Antifoam SE-15 (Sigma-Aldrich/Merck, Germany) was added. S. plymuthica WS3236 cultures were grown in terrific broth (TB) medium (Carl Roth, Germany) and modified Davis and Mingioli succinate minimal medium (succDMM: $7 \mathrm{~g} / \mathrm{L} \mathrm{K}_{2} \mathrm{HPO}_{4} * 3 \mathrm{H}_{2} \mathrm{O}, 3 \mathrm{~g} / \mathrm{L} \mathrm{KH}_{2} \mathrm{PO}_{4}, 1 \mathrm{~g} / \mathrm{L}\left(\mathrm{NH}_{4}\right)_{2} \mathrm{SO}_{4}$, $0.5 \mathrm{~g} / \mathrm{L}$ sodium citrate dihydrate, $6.49 \mathrm{~g} / \mathrm{L}$ succinate, $0.1 \mathrm{~g} / \mathrm{L} \mathrm{MgSO}_{4}{ }^{*} 7 \mathrm{H}_{2} \mathrm{O}, \mathrm{pH} 6.2$ with $\left.\mathrm{NaOH}\right)$ at $30{ }^{\circ} \mathrm{C}$ with $200 \mathrm{rpm}$ stirring. Trapping of volatile compounds started directly after inoculation and the filters were exchanged every $24 \mathrm{~h}$ for up to 4 days. $300 \mu \mathrm{L}$ of Antifoam SE-15 was again added after $40 \mathrm{~h}$ and $72 \mathrm{~h}$.

For the heterologous expression of sodorifen, E. coli BL21 cells were chemically transformed with pET28bptetO::sod_gfpv2. Expression was carried out in LB, TB and succDMM, each supplemented with $50 \mathrm{mg} / \mathrm{L}$ kanamycin. After inoculation, cells were grown at $37{ }^{\circ} \mathrm{C}$ at $200 \mathrm{rpm}$ for $5 \mathrm{~h}$ before the temperature was lowered to $16{ }^{\circ} \mathrm{C}$ and expression induced with $708 \mu \mathrm{g} / \mathrm{L}$ tetracycline. Volatile compound collection was started immediately after induction and the filters were exchanged every $24 \mathrm{~h}$ for up to 4 days. $300 \mu \mathrm{L}$ of Antifoam SE-15 was again added after $48 \mathrm{~h}$.

After fermentation, the active charcoal was extracted twice with $2 \mathrm{~mL}$ pentane, which was filtered through a $0.45 \mu \mathrm{m}$ PTFE syringe filter (Fisher Scientific, Germany) to remove remaining coal pieces before evaporation in vacuo. The residues were weighed and re-suspended in pentane or $\mathrm{CDCl}_{3}$ for further measurements. Mesitylene was used as an internal standard for GC analysis with a concentration of $250 \mu \mathrm{g} / \mathrm{mL}$.

GC-(MS) and NMR analysis of bacterial head-space extracts Analytical gas chromatography was performed at a HP 6890 Series GC (Agilent, stationary phase: HP-5 column, poly-dimethyl/diphenyl-siloxane, 95/5) with a flame ionization detector using the following temperature profile: $60{ }^{\circ} \mathrm{C}$ (hold $3 \mathrm{~min}$ ), then $15{ }^{\circ} \mathrm{C} / \mathrm{min}$ to $250{ }^{\circ} \mathrm{C}$ (hold $5 \mathrm{~min})$. The amounts of mesitylene and sodorifen were determined by peak area integration. Mass spectrometric analysis was performed with electron impact ionization (EI, $70 \mathrm{eV}$ ) on a Agilent HP 6890 Series GC-MS (Agilent, stationary phase: HP-5MS column, poly-dimethylsiloxane, 30 m, mass detection: Agilent 5973 Network Mass Selective Detector) using the same temperature profile as for analytical GC. NMR spectra of sodorifen and the $E$. coli-derived indole were directly recorded from head-space samples on a Bruker AVHD500 and a Bruker AV500-cryo spectrometer in $\mathrm{CDCl}_{3}$ (see Additional file 1: Figure S6).

\section{Additional files}

Additional file 1. Antismash and Cluster Finder results; organisation of sod-like BGCs into cluster types; phyogenetic analysis of SodC and SodD methods for the cloning and expression of the sod BGC; results of the heterologous expression of the sod cluster; GC-MS spectrum of sodorifen; NMR spectra of raw head-space samples of E. coli harbouring pET28bptetO::sod_gfpv2 expression vector.

Additional file 2. JGI whole-genome sequencing report of S. plymuthica WS3236.

\section{Abbreviations}

BGC: biosynthetic gene cluster; CTAB: cetyl trimethylammonium bromide; DiPaC: direct pathyway cloning; DXP: 1-deoxy-D-xylulose-5-phosphate; EDTA: ethylenediaminetetraacetic acid; E. coli: Escherichia coli; FPP: farnesyl pyrophosphate; GC: gas chromatography; GC-MS: gas chromatography-mass spectrometry; GFP: green fluorescent protein; IPP: isopentenyl-diphosphate; LB: lysogeny broth; MEP: non-mevalonate pathway; NMR: nuclear magnetic resonance; PCR: polymerase chain reaction; PTFE: polytetrafluoroethylene; SAM: S-adenosyl methionine; S. plymuthica: Serratia plymuthica; succDMM: modified Davis and Mingioli minimal medium containing $55 \mathrm{mM}$ succinate; TB: terrific broth; TE: Tris-EDTA; Tris: tris(hydroxymethyl)aminomethane; VOC: volatile organic compound; WGS: whole genome sequencing. 


\section{Authors' contributions}

ERD performed the cloning and expression experiments. PMD performed genomic DNA extraction and bioinformatic analysis. NS and TW performed genome sequencing. TMF contributed to strain selection and provided the $S$. plymuthica WS3236 strain. ERD, PMD and TAMG designed the overall project and wrote the manuscript. All authors edited the manuscript. All authors read and approved the final manuscript.

\begin{abstract}
Author details
${ }^{1}$ Biosystems Chemistry, Department of Chemistry and Center for Integrated Protein Science Munich (CIPSM), Technical University of Munich, Lichtenberg straße 4, 85748 Garching, Germany. ${ }^{2}$ Department of Energy, Joint Genome Institute, 2800 Mitchell Drive, Walnut Creek, CA 94598, USA. ${ }^{3}$ ZIEL Institute for Food \& Health, Lehrstuhl für Mikrobielle Ökologie, Department biowissenschaftliche Grundlagen, Technical University of Munich, Munich, Germany. ${ }^{4}$ Friedrich-Loeffler-Institut, Institut für Molekulare Pathogenese, Jena, Germany. ${ }^{5}$ Chair of Technical Biochemistry, Technische Universität Dresden, Bergstraße 66, 01602 Dresden, Germany.
\end{abstract}

\section{Acknowledgements}

We thank Andreas Tröster, Christoph Brenninger, and Simone Stegbauer (group of Prof. Dr. Thorsten Bach, TU Munich) for technical assistance with the GC and GC-MS analysis.

\section{Competing interests}

The authors declare that they have no competing interests.

\section{Availability of data and materials}

All data generated or analyzed during this study are included in this published article and its additional information files or are available from the corresponding author on reasonable request.

\section{Consent for publication}

Not applicable.

\section{Ethics approval and consent to participate}

Not applicable.

\section{Funding}

The work conducted as part of JGI Proposal \#503161 by the U.S. Department of Energy Joint Genome Institute, a DOE Office of Science User Facility, is supported under Contract No. DE-AC02-05CH11231. E.R.D. thanks the Deutsche Bundesstiftung Umwelt (DBU) for her PhD scholarship and CIPSM-Women for funding. P.M.D thanks the TUM University Foundation Fellowship and the Marie Skłodowska-Curie Actions Individual Fellowship (Project ID: 745435) for funding. We thank the DFG for generous financial support of the work in our laboratory (Emmy Noether program (GU 1233/1-1) and Center for Integrated Protein Science Munich CIPSM). This work was supported by the German Research Foundation (DFG) and the Technical University of Munich (TUM) in the framework of the Open Access Publishing Program.

\section{Publisher's Note}

Springer Nature remains neutral with regard to jurisdictional claims in published maps and institutional affiliations.

Received: 20 November 2018 Accepted: 30 January 2019

Published online: 07 February 2019

\section{References}

1. Effmert U, Kalderas J, Warnke R, Piechulla B. Volatile mediated interactions between bacteria and fungi in the soil. J Chem Ecol. 2012;38:665-703.

2. Wenke K, Kai M, Piechulla B. Belowground volatiles facilitate interactions between plant roots and soil organisms. Planta. 2010;231:499-506.

3. Wenke K, Weise T, Warnke R, Valverde C, Wanke D, Kai M, Piechulla B. Bacterial volatiles mediating information between bacteria and plants. In: Witzany G, Baluška F, editors. Biocommunication of plants. Berlin: Springer; 2012. p. 327-47.
4. Kai M, Crespo E, Cristescu SM, Harren FJ, Francke W, Piechulla B. Serratia odorifera: analysis of volatile emission and biological impact of volatile compounds on Arabidopsis thaliana. Appl Microbiol Biotechnol. 2010;88:965-76.

5. Vespermann A, Kai M, Piechulla B. Rhizobacterial volatiles affect the growth of fungi and Arabidopsis thaliana. Appl Environ Microbiol. 2007;73:5639-41.

6. Kai M, Effmert U, Berg G, Piechulla B. Volatiles of bacterial antagonists inhibit mycelial growth of the plant pathogen Rhizoctonia solani. Arch Microbiol. 2007; 187:351-60.

7. Kai M, Vespermann A, Piechulla B. The growth of fungi and Arabidopsis thaliana is influenced by bacterial volatiles. Plant Signal Behav. 2008;3:482-4.

8. Kai M, Piechulla B. Plant growth promotion due to rhizobacterial volatiles-an effect of $\mathrm{CO}_{2}$ ? FEBS Lett. 2009;583:3473-7.

9. Kai M, Piechulla B. Impact of volatiles of the rhizobacteria Serratia odorifera on the moss Physcomitrella patens. Plant Signal Behav. 2010;5:444-6.

10. von Reuss SH, Kai M, Piechulla B, Francke W. Octamethylbicyclo[3.2.1] octadienes from the rhizobacterium Serratia odorifera. Angew Chem Int Ed Engl. 2010;49:2009-10.

11. Domik D, Magnus N, Piechulla B. Analysis of a new cluster of genes involved in the synthesis of the unique volatile organic compound sodorifen of Serratia plymuthica 4Rx13. FEMS Microbiol Lett. 2016;363(14):fnw139.

12. Schmidt R, Jager V, Zuhlke D, Wolff C, Bernhardt J, Cankar K, Beekwilder J, ljcken WV, Sleutels F, Boer W, et al. Fungal volatile compounds induce production of the secondary metabolite Sodorifen in Serratia plymuthica PRI-2C. Sci Rep. 2017;7:862.

13. von Reuss S, Domik D, Lemfack MC, Magnus N, Kai M, Weise T, Piechulla B. Sodorifen biosynthesis in the rhizobacterium Serratia plymuthica involves methylation and cyclization of MEP-derived farnesyl pyrophosphate by a SAM-dependent C-methyltransferase. J Am Chem Soc. 2018;140:11855-62.

14. Magnus N, Weise T, Piechulla B. Carbon catabolite repression regulates the production of the unique volatile sodorifen of Serratia plymuthica 4Rx13. Front Microbiol. 2017;8:2522.

15. Fu J, Bian X, Hu S, Wang H, Huang F, Seibert PM, Plaza A, Xia L, Müller R, Stewart AF, Zhang Y. Full-length RecE enhances linear-linear homologous recombination and facilitates direct cloning for bioprospecting. Nat Biotechnol. 2012;30:440-6.

16. Wang H, Bian X, Xia L, Ding X, Müller R, Zhang Y, Fu J, Stewart AF. Improved seamless mutagenesis by recombineering using $\mathrm{ccdB}$ for counterselection. Nucleic Acids Res. 2014;42:e37.

17. Wang H, Li Z, Jia R, Yin J, Li A, Xia L, Yin Y, Müller R, Fu J, Stewart AF, Zhang Y. EXoCET: exonuclease in vitro assembly combined with RecET recombination for highly efficient direct DNA cloning from complex genomes. Nucleic Acids Res. 2018;46:e28.

18. Jiang W, Zhu TF. Targeted isolation and cloning of 100-kb microbial genomic sequences by Cas9-assisted targeting of chromosome segments. Nat Protoc. 2016;11:960-75.

19. Wang H, Li Z, Jia R, Hou Y, Yin J, Bian X, Li A, Müller R, Stewart AF, Fu J, Zhang $Y$. RecET direct cloning and Reda $\beta$ recombineering of biosynthetic gene clusters, large operons or single genes for heterologous expression. Nat Protoc. 2016;11:1175-90.

20. Yamanaka K, Reynolds KA, Kersten RD, Ryan KS, Gonzalez DJ, Nizet V, Dorrestein PC, Moore BS. Direct cloning and refactoring of a silent lipopeptide biosynthetic gene cluster yields the antibiotic taromycin A. Proc Natl Acad Sci USA. 2014;111:1957-62.

21. Kim JH, Feng Z, Bauer JD, Kallifidas D, Calle PY, Brady SF. Cloning large natural product gene clusters from the environment: piecing environmental DNA gene clusters back together with TAR. Biopolymers. 2010;93:833-44.

22. Kouprina $N$, Larionov $V$. Selective isolation of genomic loci from complex genomes by transformation-associated recombination cloning in the yeast Saccharomyces cerevisiae. Nat Protoc. 2008;3:371-7.

23. Quan J, Tian J. Circular polymerase extension cloning for high-throughput cloning of complex and combinatorial DNA libraries. Nat Protoc. 2011;6:242-51.

24. Zeng F, Zang J, Zhang S, Hao Z, Dong J, Lin Y. AFEAP cloning: a precise and efficient method for large DNA sequence assembly. BMC Biotechnol. 2017;17:81. 
25. Greunke C, Duell ER, D’Agostino PM, Glöckle A, Lamm K, Gulder TAM. Direct pathway cloning ( $\mathrm{DiPaC})$ to unlock natural product biosynthetic potential. Metab Eng. 2018;47:334-45.

26. D'Agostino PM, Gulder TAM. Direct pathway cloning combined with sequence- and ligation-independent cloning for fast biosynthetic gene cluster refactoring and heterologous expression. ACS Synth Biol. 2018;7:1702-8

27. Blin K, Wolf T, Chevrette MG, Lu X, Schwalen CJ, Kautsar SA, Suarez Duran HG, de Los Santos ELC, Kim HU, Nave M, et al. antiSMASH 4.0-improvements in chemistry prediction and gene cluster boundary identification. Nucleic Acids Res. 2017:45:W36-41.

28. Cimermancic P, Medema MH, Claesen J, Kurita K, Wieland Brown LC, Mavrommatis K, Pati A, Godfrey PA, Koehrsen M, Clardy J, et al. Insights into secondary metabolism from a global analysis of prokaryotic biosynthetic gene clusters. Cell. 2014;158:412-21.

29. Masschelein J, Mattheus W, Gao LJ, Moons P, Van Houdt R, Uytterhoeven B, Lamberigts C, Lescrinier E, Rozenski J, Herdewijn P, et al. A PKS/NRPS/ FAS hybrid gene cluster from Serratia plymuthica RVH1 encoding the biosynthesis of three broad spectrum, zeamine-related antibiotics. PLoS ONE. 2013:8:e54143.

30. Ongley SE, Bian X, Zhang Y, Chau R, Gerwick WH, Müller R, Neilan BA. High-titer heterologous production in E. coli of lyngbyatoxin, a protein kinase $C$ activator from an uncultured marine cyanobacterium. ACS Chem Biol. 2013;8:1888-93.

31. Elgaali H, Hamilton-Kemp TR, Newman MC, Collins RW, Yu K, Archbold DD. Comparison of long-chain alcohols and other volatile compounds emitted from food-borne and related Gram positive and Gram negative bacteria. J Basic Microbiol. 2002;42:373-80

32. Yu K, Hamilton-Kemp TR, Archbold DD, Collins RW, Newman MC. Volatile compounds from Escherichia coli $0157: \mathrm{H} 7$ and their absorption by strawberry fruit. J Agric Food Chem. 2000;48:413-7.
33. Liu T, Mazmouz R, Ongley SE, Chau R, Pickford R, Woodhouse JN, Neilan $B A$. Directing the heterologous production of specific cyanobacterial toxin variants. ACS Chem Biol. 2017:12:2021-9.

34. Li P, Kwok AH, Jiang J, Ran T, Xu D, Wang W, Leung FC. Comparative genome analyses of Serratia marcescens FS14 reveals its high antagonistic potential. PLoS ONE. 2015;10:e0123061.

35. Micallef ML, Sharma D, Bunn BM, Gerwick L, Viswanathan R, Moffitt MC. Comparative analysis of hapalindole, ambiguine and welwitindolinone gene clusters and reconstitution of indole-isonitrile biosynthesis from cyanobacteria. BMC Microbiol. 2014;14:213.

36. Eid J, Fehr A, Gray J, Luong K, Lyle J, Otto G, Peluso P, Rank D, Baybayan $P$, Bettman $B$, et al. Real-time DNA sequencing from single polymerase molecules. Science. 2009;323:133-8.

37. Chin CS, Alexander DH, Marks P, Klammer AA, Drake J, Heiner C, Clum A, Copeland A, Huddleston J, Eichler EE, et al. Nonhybrid, finished microbial genome assemblies from long-read SMRT sequencing data. Nat Methods. 2013;10:563-9

38. Chen IMA, Chu K, Palaniappan K, Pillay M, Ratner A, Huang J, Huntemann M, Varghese N, White JR, Seshadri R, et al. IMG/M v.5.0: an integrated data management and comparative analysis system for microbial genomes and microbiomes. Nucleic Acids Res. 2018;47:D666-77.

39. Dereeper A, Audic S, Claverie JM, Blanc G. BLAST-EXPLORER helps you building datasets for phylogenetic analysis. BMC Evol Biol. 2010;10:8.

40. Dereeper A, Guignon V, Blanc G, Audic S, Buffet S, Chevenet F, Dufayard JF, Guindon S, Lefort V, Lescot M, et al. Phylogeny.fr: robust phylogenetic analysis for the non-specialist. Nucleic Acids Res. 2008;36:W465-9.
Ready to submit your research? Choose BMC and benefit from:

- fast, convenient online submission

- thorough peer review by experienced researchers in your field

- rapid publication on acceptance

- support for research data, including large and complex data types

- gold Open Access which fosters wider collaboration and increased citations

- maximum visibility for your research: over $100 \mathrm{M}$ website views per year

At $\mathrm{BMC}$, research is always in progress.

Learn more biomedcentral.com/submissions 